\title{
Modeling of a Plasma Antenna with Inhomogeneous Distribution of Electron Density
}

\author{
Zong-sheng Chen, ${ }^{1}$ Li-fang $\mathrm{Ma}^{2}{ }^{2}$ and Jia-chun Wang ${ }^{1}$ \\ ${ }^{1}$ State Key Laboratory of Pulsed Power Laser Technology, Electronic Engineering Institute, Hefei, Anhui 230037, China \\ ${ }^{2}$ Institute of Information Ammunition, Academy of Army Officers, Hefei, Anhui 230031, China \\ Correspondence should be addressed to Zong-sheng Chen; chenzongsh12@163.com
}

Received 16 October 2014; Accepted 26 January 2015

Academic Editor: Atsushi Mase

Copyright (c) 2015 Zong-sheng Chen et al. This is an open access article distributed under the Creative Commons Attribution License, which permits unrestricted use, distribution, and reproduction in any medium, provided the original work is properly cited.

\begin{abstract}
The distribution of the electron density along a plasma antenna can influence the antenna's performance. But little has been done in this regard in former studies. In this paper, a model of a practical plasma antenna with an inhomogeneous distribution of electron density is founded according to the transmission-line equivalent theory of a metal monopole, from which the current distribution and the radiation pattern of a plasma antenna with appropriate parameters are calculated. The results show that the electrical current distribution, the maximum radiation direction, and the beamwidth of a plasma antenna vary with electron density distributions. To validate the model, the plasma antenna with the same parameters is also simulated based on electromagnetic software HFSS. It is found that the results from the two ways are almost consistent.
\end{abstract}

\section{Introduction}

Compared with the conventional metal antenna, plasma antennas use ionized gas as the conducting medium instead of metal, which have excellent potentials for applications and have attracted intensive interest of researchers [1-3]. Models of the plasma antenna and simulations of the radiation pattern by using different numerical methods have been provided [4-7], and some significative results have been achieved. A number of the models were founded on the dispersion relation of surface wave along ideal plasma columns in vacuum $[1,4-6]$. But for practical plasma antennas, the plasma is restricted in a tube. So the outer and inner radius of the tube, the dielectric constant of the tube material, and the density distribution of the plasma should be also taken into account in the computation. Zhao et al. [7] examined influences of the dielectric constant and the radius of the tube on the far-field radiation pattern of the plasma antenna. However, the model assumed that the density distribution of the plasma in the tube is homogeneous, other than the linear distribution in the actual tube $[3,8]$. In the present paper, we developed a model of the plasma antenna with inhomogeneous distribution of the electron density. From the model, electrical current distributions and radiation patterns of plasma antennas can be calculated. Moreover, another model of the plasma antenna was constructed using electromagnetic software HFSS, which is an interactive software package for calculating the electromagnetic behavior of a structure [9]. Results from the software could attest the validity of the first model.

\section{Theoretical Model}

The electrical current distribution of the plasma antenna can be expressed mathematically and analogously to that of a monopole. According to the transmission-line equivalent theory of a metal monopole, the current distribution along the antenna can be written as

$$
\begin{aligned}
I(z)=I_{0} \times & {\left[e^{-i \int_{0}^{z} k_{r} d z^{\prime}} \times e^{-\int_{0}^{z} k_{i} d z^{\prime}}\right.} \\
& \left.-e^{-i\left(2 \int_{0}^{L} k_{r} d z^{\prime}-\int_{0}^{z} k_{r} d z^{\prime}\right)} \times e^{-\left(2 \int_{0}^{L} k_{i} d z^{\prime}-\int_{0}^{z} k_{i} d z^{\prime}\right)}\right],
\end{aligned}
$$


where $I_{0}$ is the magnitude of the current, $L$ is the length of the antenna, $z$ is the axial distance from the bottom of the antenna, $k_{r}$ is the real part of the wave vector $k(z)$, while $k_{i}$ is the imaginary part.

For a metal monopole, the far-field radiation is well known as

$$
F(\theta)=\left|\frac{\sin (\theta)}{I_{\max }} \int_{-L}^{L} I\left(z^{\prime}\right) \exp \left(j k_{0} z^{\prime} \cos (\theta)\right) d z^{\prime}\right|,
$$

where $I_{\max }$ is the maximal value of the current on the antenna.

Using (1) and (2), the normalized radiation pattern of the plasma antenna can be calculated if the wave vector $k(z)$ is known.

For a practical plasma antenna, the plasma column of a radius $R$ is contained in a dielectric tube of a thickness $d$. A communication signal with an angular frequency $\omega$ is launched at one end of the antenna. The electron plasma angular frequency is $\omega_{p}$. If $\omega_{p}>\omega$, the communication signal propagates as a surface wave. There have been many researches on the surface wave both theoretically and experimentally [8]. IVAN and EVGHENIA investigated its characteristics detailedly when they examined plasma resources produced and sustained by a travelling electromagnetic surface wave [10]. From their study, provided that the plasma density is radially constant, the dispersion relation of the surface wave along a plasma column can be written as

$$
\frac{\varepsilon_{p}}{a_{p}} \frac{I_{1}\left(a_{p}\right)}{I_{0}\left(a_{p}\right)}+\frac{\varepsilon_{d}}{a_{d}} \frac{\left[a_{1}+a_{2} a_{d} K_{1}\left(\gamma a_{v}\right) / \varepsilon_{d} a_{v} K_{0}\left(\gamma a_{v}\right)\right]}{\left[a_{3}+a_{4} a_{d} K_{1}\left(\gamma a_{v}\right) / \varepsilon_{d} a_{v} K_{0}\left(\gamma a_{v}\right)\right]}=0,
$$

where

$$
\begin{gathered}
a_{1}=J_{1}\left(a_{d}\right) H_{1}^{(1)}\left(\gamma a_{d}\right)-H_{1}^{(1)}\left(a_{d}\right) J_{1}\left(\gamma a_{d}\right), \\
a_{2}=J_{1}\left(a_{d}\right) H_{0}^{(1)}\left(\gamma a_{d}\right)-H_{1}^{(1)}\left(a_{d}\right) J_{0}\left(\gamma a_{d}\right), \\
a_{3}=H_{0}^{(1)}\left(a_{d}\right) J_{1}\left(\gamma a_{d}\right)-J_{0}\left(a_{d}\right) H_{1}^{(1)}\left(\gamma a_{d}\right), \\
a_{4}=H_{0}^{(1)}\left(a_{d}\right) J_{0}\left(\gamma a_{d}\right)-J_{0}\left(a_{d}\right) H_{0}^{(1)}\left(\gamma a_{d}\right), \\
\varepsilon_{p}=1-\frac{\omega_{p}^{2}}{\omega(\omega+i v)}
\end{gathered}
$$

and $a_{p}=\sqrt{x^{2}-\sigma^{2} \varepsilon_{p}}, a_{d}=\sqrt{\sigma^{2} \varepsilon_{d}-x^{2}}, a_{v}=\sqrt{x^{2}-\sigma^{2}}$, $x=k R, \sigma=\omega R / c, \gamma=1+d / R, v$ is the collision frequency of plasma, $J_{0}$ and $J_{1}$ are the Bessel functions, $I_{0}, I_{1}, K_{0}$, and $K_{1}$ are the modified Bessel functions, $H_{0}^{(1)}$ and $H_{1}^{(1)}$ are the Hankel functions, and $\varepsilon_{d}$ is the dielectric constant of the tube material.

If we assume $d=0$, (3) becomes

$$
\frac{\varepsilon_{p} T_{0} I_{1}\left(T_{p} R\right)}{K_{1}\left(T_{0} R\right)}+\frac{T_{p} I_{0}\left(T_{p} R\right)}{K_{0}\left(T_{0} R\right)}=0,
$$

where $T_{p}^{2}=k^{2}-\varepsilon_{p} k_{0}^{2}, T_{0}^{2}=k^{2}-k_{0}^{2}, k_{0}=\omega / c$. Equation (5) is just the dispersion relation of surface wave along plasma column in vacuum as shown in former studies [4-6].

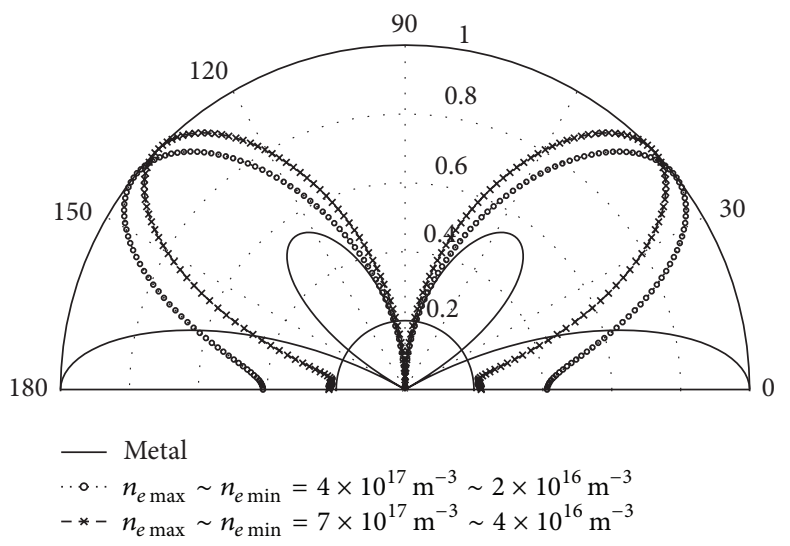

FIGURE 1: Normalized radiation pattern of the plasma antenna.

The electron density along the plasma antenna decreases almost linearly and can be expressed approximately as $[3,8$, 11]

$$
n_{e}(z)=n_{e \max }+\frac{z}{L}\left(n_{e \min }-n_{e \max }\right),
$$

where $n_{e \max }$ is the density at the bottom of the plasma antenna while $n_{e \min }$ is that at the top.

\section{Numerical Results and Discussion}

Using (1) to (3) and (6), the normalized radiation pattern of the plasma antenna and its electrical current distribution were calculated with $R=12.5 \mathrm{~mm}, 2 \times 10^{16} \mathrm{~m}^{-3} \leq n_{e \min } \leq 4 \times$ $10^{16} \mathrm{~m}^{-3}, 4 \times 10^{17} \mathrm{~m}^{-3} \leq n_{e \max } \leq 7 \times 10^{17} \mathrm{~m}^{-3}, \varepsilon_{d}=3.78, L=$ $1 \mathrm{~m}, v=4 \times 10^{8} \mathrm{~Hz}, d=0.9 \mathrm{~mm}$, and a communication signal frequency $f=200 \mathrm{MHz}$. These parameters are consistent with the cases of our experiments, which have been proven reasonable for the practical plasma antenna. With other appropriate values, the model founded in our paper can be also applicable. Figure 1 is the normalized radiation pattern of the plasma antenna with different distributions of electron density. Comparatively, the normalized radiation pattern of a metallic antenna with the same length is also presented. The calculating results show that the normalized radiation pattern of the plasma antenna is markedly influenced by the electron density distribution and has great difference from that of a metallic antenna with the same length. In spite of the same collision frequency and length, the maximum radiation direction and beamwidth of the plasma antenna vary due to different density distributions. The main reason for this phenomenon is that the plasma density and its collision frequency determine the wave vector $k$, which impacts the electrical current distribution along the plasma antenna. Figure 2 gives the real part and the imaginary part of the wave vector $k$ along the plasma antenna.

From Figure 2, we can find that $\operatorname{Re}(k)$ and $\operatorname{Im}(k)$ have great changes axially, which result from electron density distributions. On the contrary, if we assume that the plasma density is uniform, then the value of the wave vector $k$ is a fixed value, which deviates from the fact. From the bottom to 


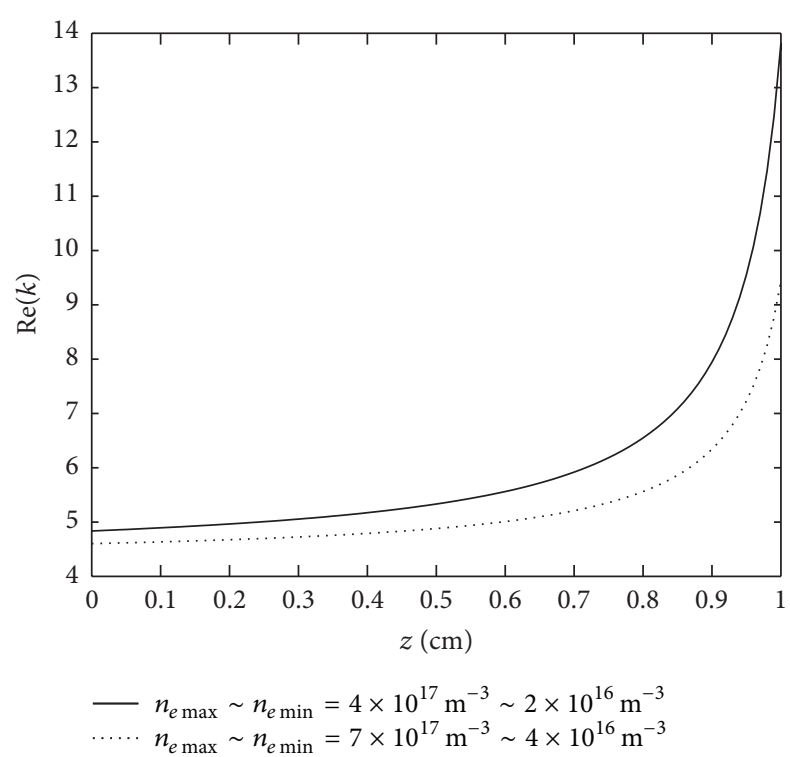

(a)

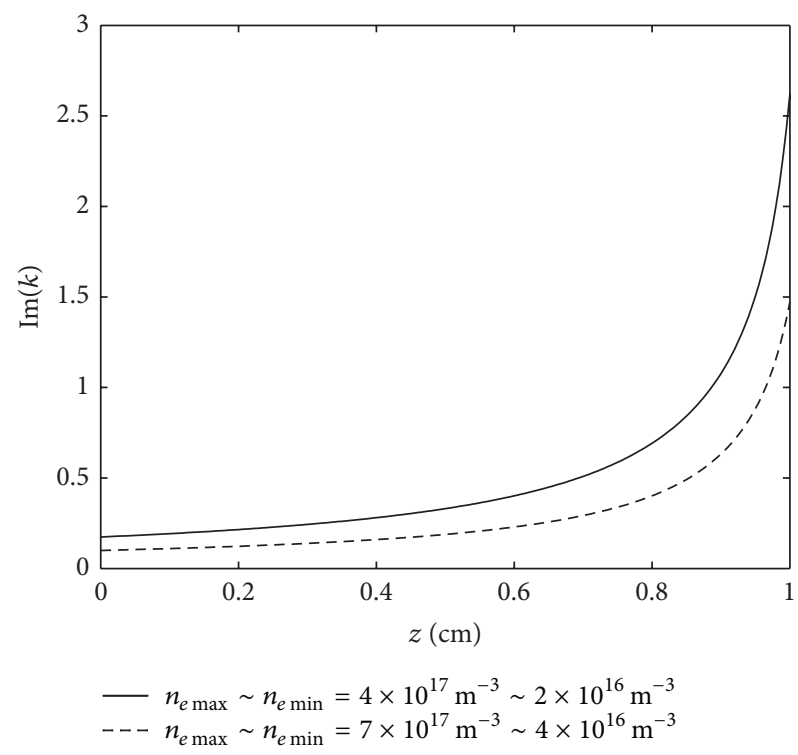

(b)

FIGURE 2: Real part and imaginary part of the wave vector $k$ along the plasma antenna.

the top of the plasma antenna, $\operatorname{Im}(k)$ increases with decline of the plasma density. This indicates that attenuation of the signal augments, so the plasma antenna can be viewed as a type of antenna with a continuously varying resistive loading. Additionally, under the condition of an invariable collision frequency, $\operatorname{Re}(k)$ and $\operatorname{Im}(k)$ both decrease along with the increase of the plasma density. Figure 3 is the current distribution along the plasma antenna, which shows that the density distribution along the plasma antenna influences the electrical current distribution. Actually, it is because different electrical current distributions lead to variations of the maximum radiation direction and the beamwidth.

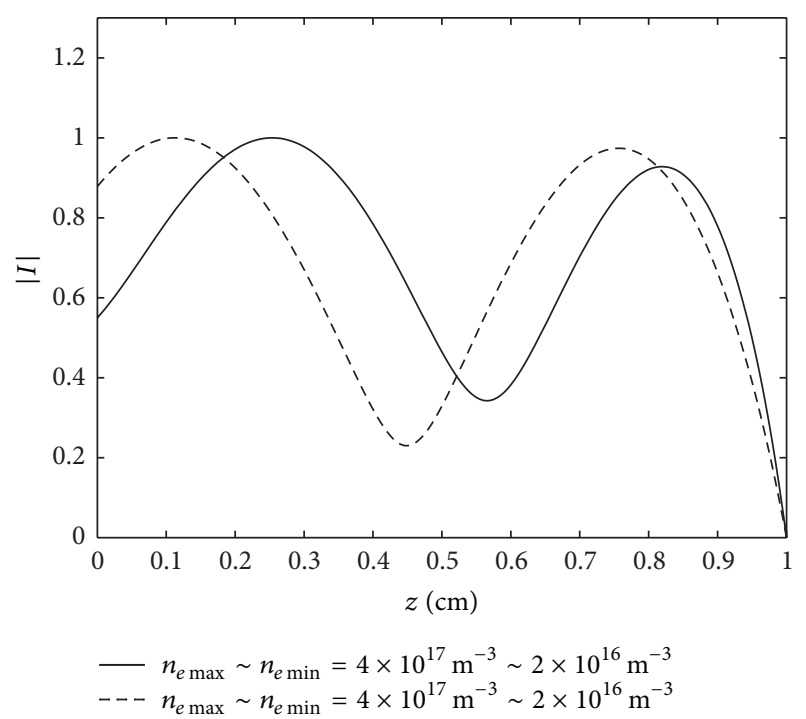

FIGURE 3: Current distribution along the plasma antenna.

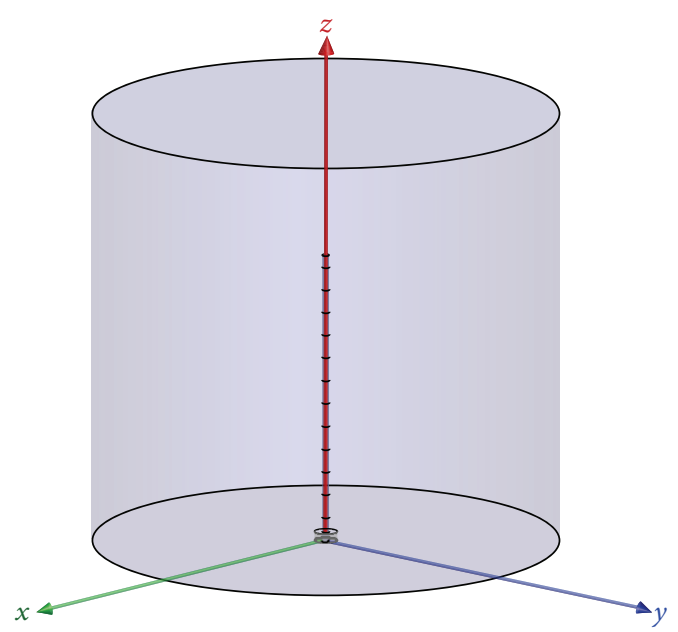

FIGURE 4: HFSS model of the plasma antenna with 13 segments.

Moreover, we found another model of the plasma antenna based on HFSS, which is a useful tool in the field of antenna design. When setting up the model, we should pay attention to the dielectric constant and the conductivity of the plasma, because they are both involved with the electron density, the collision frequency, and the communication signal frequency. For the plasma antenna with the liner distribution of electron density, its dielectric constant and conductivity vary axially even with the same communication signal frequency. Here, to be convenient for simulation, the plasma antenna is divided into sufficient segments, in which the electron density is assumed uniform. As a result, the dielectric constants and conductivities of adjacent segments will have a little discrepancy. These settings will benefit the veracity of the simulation. Figure 4 is the HFSS model of the plasma antenna, which is put on an infinite metallic plane. The air volume object is defined as a radiation boundary, at which waves are absorbed completely, essentially ballooning the boundary infinitely far 


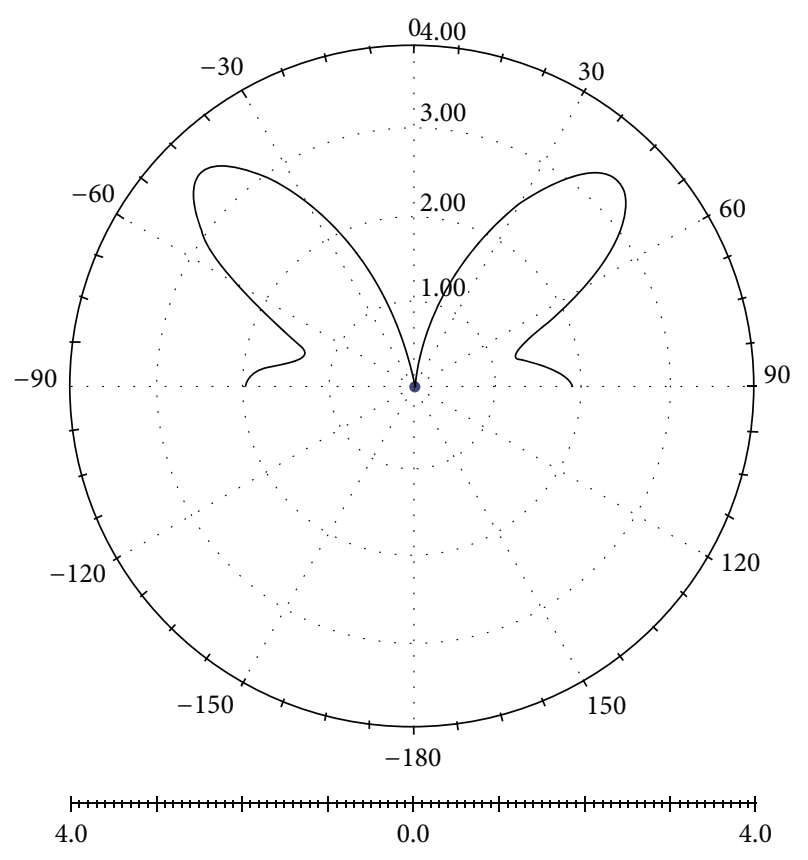

FIGURE 5: Radiation pattern of the plasma antenna from HFSS model.

away from the antenna. At the end of the antenna, a lumped port is assigned.

To compare with the first model and its calculating results, the same parameters are set in the HFSS model. The plasma antenna is divided into 13 segments. The length of the first 12 segments from the bottom is $8 \mathrm{~cm}$ each while that of the last is $4 \mathrm{~cm}$. We find that results acquired from the model with 13 segments are almost the same as those from the model with 20 segments, so 13 segments are enough for our simulation. Figure 5 gives the radiation pattern of the plasma antenna simulated with $n_{e \min }=2 \times 10^{16} \mathrm{~m}^{-3}$ and $n_{e \max }=4 \times 10^{17} \mathrm{~m}^{-3}$. Its electrical current distribution is shown in Figure 6. From Figures 1, 3, 5, and 6, we can get that the results of the two models are almost consistent, which indicates that both models are valid.

\section{Conclusions}

Taking the electron density distribution of plasma into account, a model of the practical plasma antenna is founded, from which we can calculate electrical current distributions and radiation patterns of plasma antennas. The results calculated with appropriate parameters show that the electrical current distribution, the maximum radiation direction, and the beamwidth of the plasma antenna vary due to different density distributions. As for the plasma antenna, the electron density distribution is vitally important to its performance. Different from the case of the uniform electron density, signal attenuation augments due to the linear decline of the plasma density from the bottom to the top, so the plasma antenna can be viewed as a type of antenna with a continuously varying resistive loading. Because the model only needs the condition

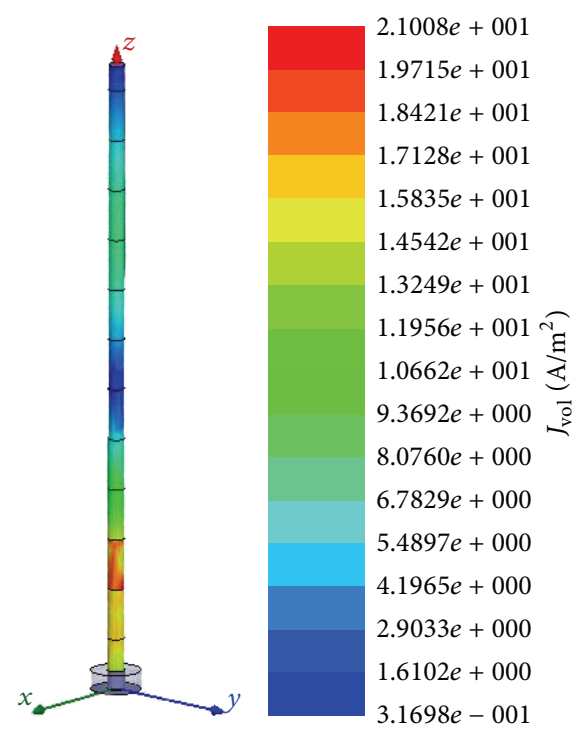

FIGURE 6: Electrical current distribution along the plasma antenna from HFSS model.

that a communication signal must propagate as a surface wave along the plasma antenna, the results from the model can be extended to other communication signal frequencies if the electron plasma angular frequency is higher than the communication signal angular frequency. The results from the model we set up in this paper are in close agreement with those from the HFSS model. This model seems useful in studying the plasma antenna.

\section{Conflict of Interests}

The authors declare that there is no conflict of interests regarding the publication of this paper.

\section{Acknowledgment}

This work is supported by the Fund of National Defense PreResearch (Grant no. 07048).

\section{References}

[1] G. G. Borg, J. H. Harris, D. G. Miljak, and N. M. Martin, "Application of plasma columns to radio frequency antennas," Applied Physics Letters, vol. 74, no. 22, pp. 3272-3274, 1999.

[2] I. Alexeff, T. Anderson, S. Parameswaran, E. P. Pradeep, J. Hulloli, and P. Hulloli, "Experimental and theoretical results with plasma antennas," IEEE Transactions on Plasma Science, vol. 34, no. 2, pp. 166-172, 2006.

[3] S. Wang, N. Sun, J. Li, Q. Xiang, and C. Wei, "Essential characteristics of plasma antennas driven by one-ended surface wave," Plasma Science and Technology, vol. 12, no. 2, pp. 230$234,2010$.

[4] Y. Lee and S. Ganguly, "Analysis of a plasma-column antenna using FDTD method," Microwave and Optical Technology Letters, vol. 46, no. 3, pp. 252-259, 2005. 
[5] J. P. Rayner, A. P. Whichello, and D. Cheetham, "Physical characteristics of plasma antennas," IEEE Transactions on Plasma Science, vol. 32, no. 1, pp. 269-281, 2004.

[6] Z. H. Qian, R. S. Chen, H. W. Yang, K. W. Leung, and E. K. N. Yung, "FDTD analysis of a plasma WHIP antenna," Microwave and Optical Technology Letters, vol. 47, no. 2, pp. 147-150, 2005.

[7] G. W. Zhao, Y.-M. Xu, and C. Chen, "Calculation of dispersion relation and radiation pattern of plasma antenna," Acta Physica Sinica, vol. 56, no. 9, pp. 5298-5303, 2007 (Chinese).

[8] M. Moisan and Z. Zakrzewski, "Plasma sources based on the propagation of electromagnetic surface waves," Journal of Physics D: Applied Physics, vol. 24, no. 7, pp. 1025-1048, 1991.

[9] Ansoft HFSS Online Help, http://ansoft.com/.

[10] I. Zhelyazkov and E. Benova, "Modeling of a plasma column produced and sustained by a traveling electromagnetic surface wave," Journal of Applied Physics, vol. 66, no. 4, pp. 1641-1650, 1989.

[11] A. Sola, J. Cotrino, A. Gamero, and V. Colomer, "Study of surface-wave-produced plasma column lengths," Journal of Physics D: Applied Physics, vol. 20, no. 10, pp. 1250-1258, 1987. 

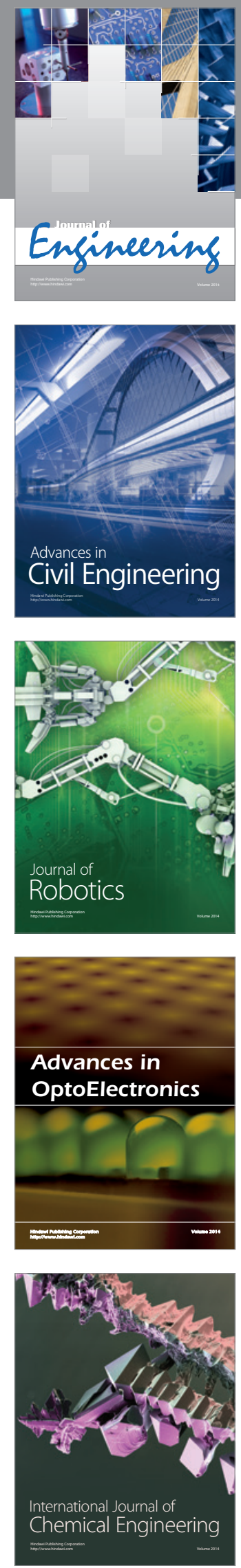

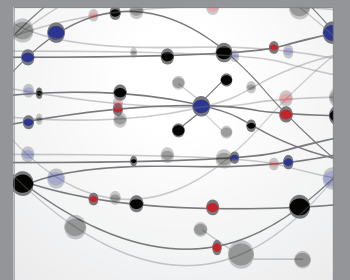

The Scientific World Journal
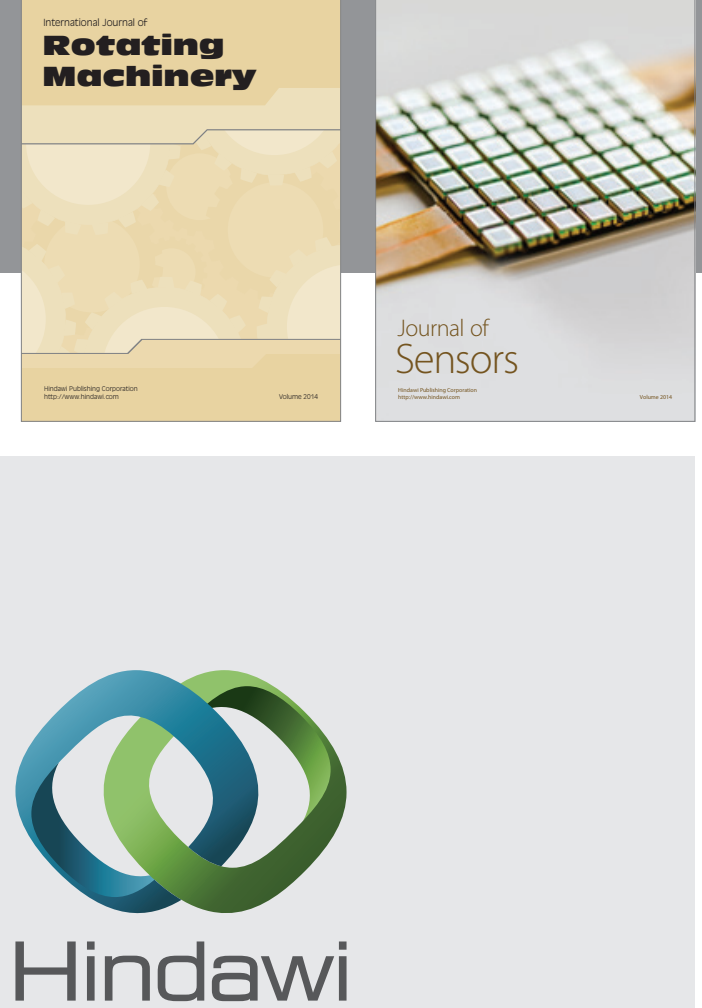

Submit your manuscripts at http://www.hindawi.com
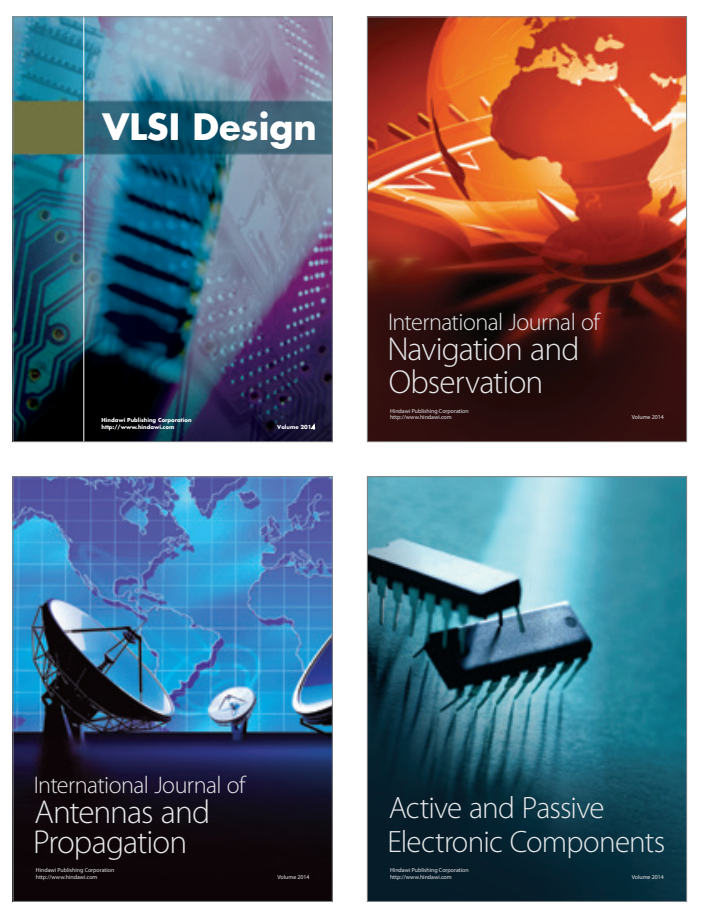
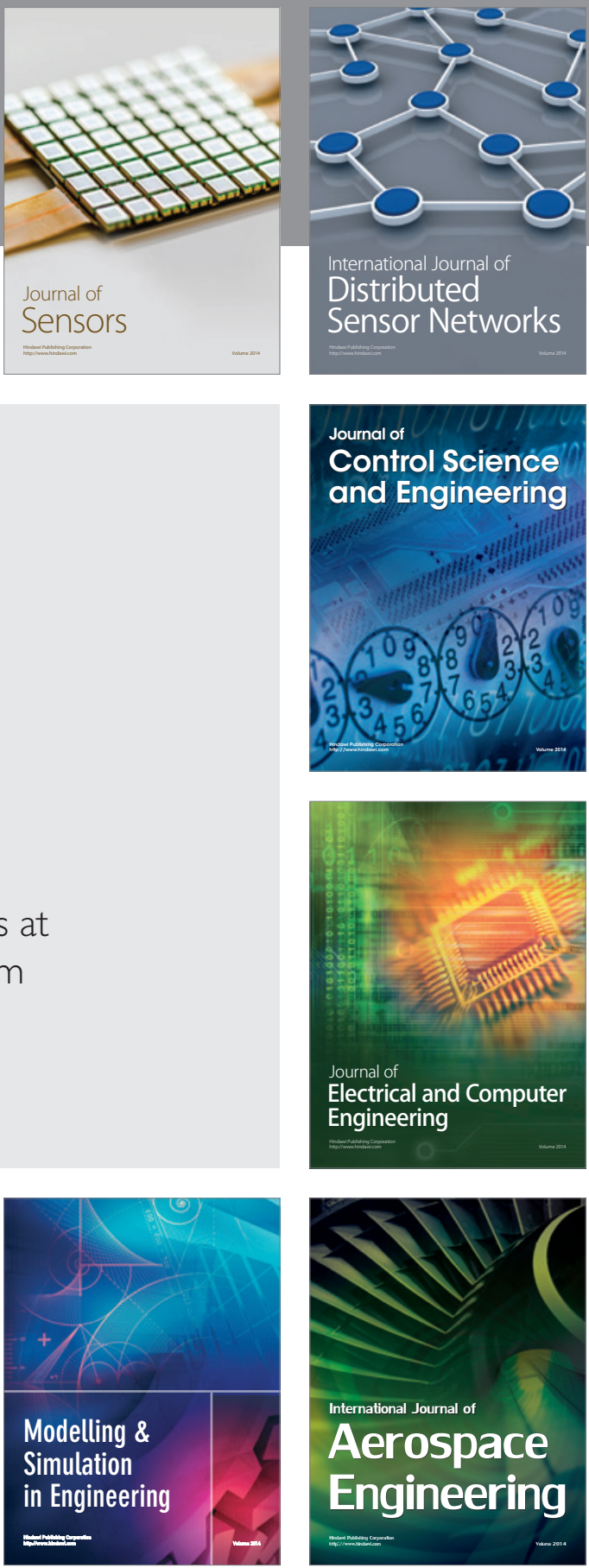

Journal of

Control Science

and Engineering
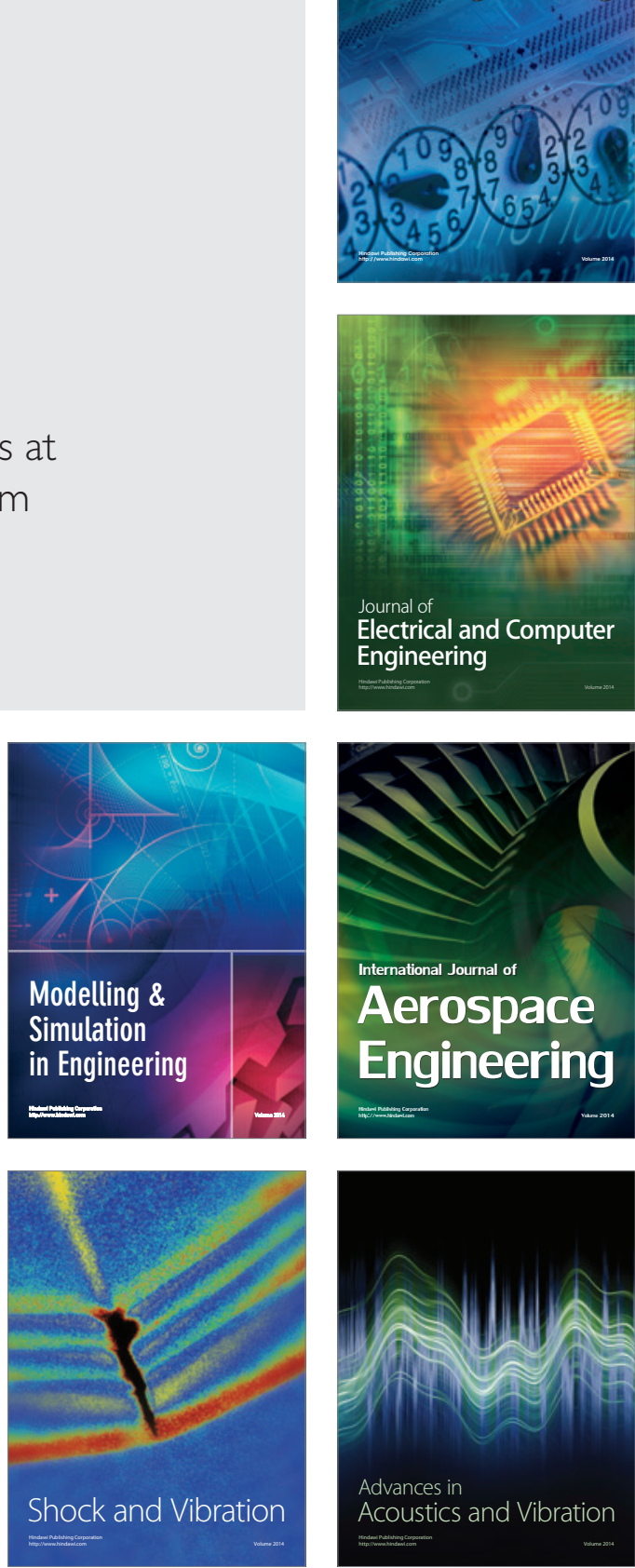